\title{
Welcome to the animal pharmaceuticals special focus
}

\author{
"The articles in this mini focus provide a glimpse into the evolving challenges influencing the \\ development and use of veterinary drug products."
}

To date, many of the leading pharmaceutical journals have predominantly focused on issues directly related to human drug development. Lack of attention to veterinary medicine reflects the difficulty in communicating universal lessons learned from the successes, failures and roadblocks we have encountered. From exploring the attributes of drug-delivery platforms to the assessment of novel therapeutic targets, the animal patient provides an invaluable model for human pharmaceuticals. For example, there are shared challenges in targeted drug delivery (e.g., due to transporters, immune responses and physical barriers). There is commonality in the issues associated with the assessment of pain amelioration in neonates, comatose patients and veterinary species. In addition, there is a tremendous amount of information being harnessed by studying the relatively homogenous DNA sequences of pure-bred animals in order to identify the genetic basis of diseases that are common to animals and humans [101].

Issues confronted in the development of veterinary tablet formulations are consistent with those encountered in human medicine [1]. In this regard, the fundamental difference between human and veterinary formulations pertain to the drug-release characteristics required to accommodate the physiological idiosyncrasies of the animal patient [2-4]. We rarely consider the possibility that species-specific differences in product performance could provide insights into some of the problems that arise in human medicine. For example, could the failure of some oral formulations to perform in the dog (due to such physiological differences as their higher gastric $\mathrm{pH}$, leakier intestinal membranes and faster intestinal transit time) reflect potential formulation failures that can occur when tablets are administered to human patients presenting with gastrointestinal abnormalities such as achlorhydria, hypermotility or intestinal enteropathies?
The changing needs associated with animal therapeutics mirrors the evolution of human society. The dwindling number of small familyowned farms and the growing predominance of consolidated facilities have led to a demand for drugs that can maximize animal growth and feed efficiency, synchronize estrus and control the outbreak of disease. Even the nutritional composition of animal-derived tissues has come under scrutiny as consumers seek to purchase leaner cuts of meat. For companion animal species, the pet has an increasingly important role within the nuclear family. Consequently, there is a need for treatments that target diseases such as age-related pathologies (many of which parallel those encountered in human medicine), cancer, heart disease, endocrine disorders, behavioral disorders and parasitic and microbial infections.

\section{"Issues confronted in the development of veterinary tablet formulations are consistent with those encountered in human medicine."}

Attention is being directed towards formulating products for ease of administration in order to encourage owner compliance and minimize animal stress. For food-producing animals, there is the additional concern of human food safety and the need to avoid anything that could compromise the environment. These multifaceted demands are being addressed through the development of novel active pharmaceutical ingredients, drug combinations and drug-delivery platforms. These innovations raise the issue of patent law, which is discussed in this issue by Lu et al. [5].

One of the drug classes that has been a candidate for novel extended-release formulations and drug combinations is the antiparasitic class. These compounds are highly toxic to the parasite but must be safe to the veterinary recipient. There is little margin for adverse reactions

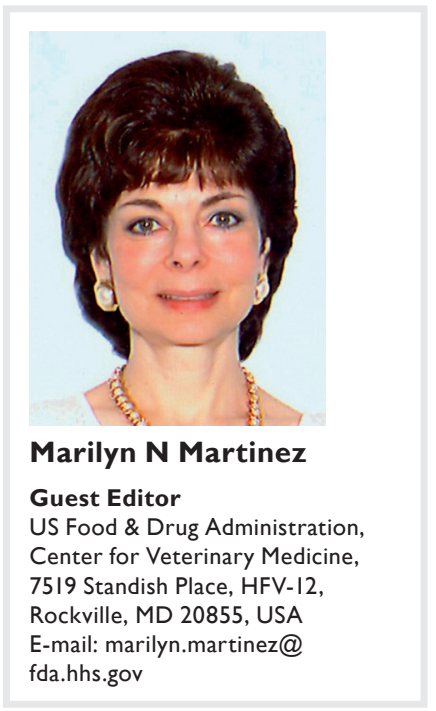

FUTURE
SCIENCE FSS 
since these products are usually administered to healthy animals. There can be no risk to human health upon direct contact with the treated animal. Convenience of application is also essential to ensure owner compliance, since there may not be the dosing incentive that exists when treating an existing disease. In this issue, Woods and colleagues provide an overview of their experiences of antiparasitic drug development within Pfizer Animal Health.

Riviere noted that changing needs will likely drive veterinary medicine towards high-technology options, such as large-molecule therapies, novel targeted drug-delivery platforms and nanotechnology and implantable physiological feedback systems [6]. In the development of these products, the paradigms previously applied to the assessment of product safety and effectiveness for small molecules may need to be revised. Hunter presents an overview of the current status of animal health drug development [7]. In a similar vein, Schofield provides a synopsis of how target animal safety is currently being evaluated for veterinary dosage forms [8].

There is a growing need to identify those factors that can influence population dose-exposure-response relationships. In this regard, variations in the activity of drug-metabolizing enzymes can have a tremendous impact on in vivo product performance. The number of complex molecules entering the veterinary pharmaceutical pipeline underscores the importance of identifying variables that can influence systemic exposure to the parent compound and any active metabolite. This topic is discussed by Antonovic and Martinez [9].
As we progress towards the future, there will be an increasing emphasis on understanding the drug target, the drug molecule and the interplay between host physiology and dosage form performance. It will be important to understand how we can use prognostic tools and readily identifiable covariates (e.g., age, gender, disease and breed) to predict therapeutic outcomes. Optimally, these predictive methodologies will be readily available and promote cost-effective product development. To this end, it is essential that the veterinary pharmaceutical community embraces many of the innovations being explored by our human health counterparts. Similarly, we need to reach out to our human health colleagues so that they too can benefit from the invaluable lessons learned through our product development experiences. Ultimately, forward progress will depend upon our ability to foster an environment that encourages drug companies to make the investments necessary to develop these much-needed veterinary drug products. We are certainly well on the road to making all of these goals a reality.

We invite all readers to enjoy this special focus!

\section{Financial \& competing interests disclosure}

The author has no relevant affliations or financial involvement with any organization or entity with a financial interest in or financial conflict with the subject matter or materials discussed in the manuscript. This includes employment, consultancies, honoraria, stock ownership or options, expert testimony, grants or patents received or pending, or royalties.

No writing assistance was utilized in the production of this manuscript.

\section{Bibliography}

1 Fahmy R, Danielson D, Martinez M. Formulation and design of veterinary tablets. In: Pharmaceutical Dosage Forms: Tablets (3rd Edition). Augsburger LL, Hoag SW (Eds). Informa Healthcare Inc., NY, USA, 383-431 (2008).

2 Martinez M, Amidon G, Clarke L, Jones WW, Mitra A, Riviere J. Applying the biopharmaceutics classification system to veterinary pharmaceutical products. Part II. Physiological considerations. Adv. Drug Deliv. Rev. 54(6), 825-850 (2002).

3 Martinez MN, Papich MG. Factors influencing the gastric residence of dosage forms in dogs. J. Pharm. Sci. 98(3), 844-860 (2009).
4 Rathbone MJ, Martinez MN. Modified release drug delivery in veterinary medicine. Drug Discov. Today 7(15), 823-829 (2002).

5 Lu DL, Kowalski TJ, Jarecki-Black J. Intellectual property issues for veterinary pharmaceuticals in the USA. Future Med. Chem. 3(7), 847-850 (2011).

6 Riviere JE. The future of veterinary therapeutics: a glimpse towards 2030. Vet. J. 174(3), 462-471 (2007).

7 Hunter RP, Shryock TR, Cox BR, Butler RM, Hammelman JE. Overview of the animal health drug development and registration process: an industry perspective. Future Med. Chem. 3(7), 881-886 (2011).
8 Schofield J. Animal-health pharmaceuticals: research responsibilities and efforts in target animal safety and laboratory animal welfare. Future Med. Chem. 3(7), 851-854 (2011).

9 Martinez M, Antonovic L. Role of the cytochrome P450 enzyme system in veterinary pharmacokinetics: where are we now? Where are we going? Future Med. Chem. 3(7), 855-879 (2011).

\section{- Website}

101 Dog genome resources. www.ncbi.nlm.nih.gov/genome/guide/dog/ (Accessed 24 February 2011) 